\title{
Integration of the blood test into the low-dose computed tomography lung cancer screening: reliable discrimination between malignant and non-malignant radiographic findings
}

\author{
Ekaterina S. Kuligina ${ }^{1,2}$, Aglaya G. Iyevleva ${ }^{1,2}$, Evgeny N. Imyanitov ${ }^{1,2,3}$ \\ ${ }^{1}$ N.N. Petrov Institute of Oncology, St.-Petersburg, Russia; ${ }^{2}$ St.-Petersburg Pediatric Medical University, St.-Petersburg, Russia; ${ }^{3}$ I.I. Mechnikov \\ North-Western Medical University, St.-Petersburg, Russia \\ Correspondence to: Ekaterina S. Kuligina. N.N. Petrov Institute of Oncology, Leningradskaya str. 68, Pesochny, St.-Petersburg 197758, Russia. \\ Email: kate.kuligina@gmail.com. \\ Comment on: Liang W, Chen Z, Li C, et al. Accurate diagnosis of pulmonary nodules using a noninvasive DNA methylation test. J Clin Invest \\ 2021;131:145973.
}

Submitted Aug 31, 2021. Accepted for publication Sep 24, 2021.

doi: $10.21037 /$ tlcr-21-680

View this article at: https://dx.doi.org/10.21037/tlcr-21-680

Lung cancer (LC) is the most common oncological disease worldwide. Its incidence currently exceeds 2 million cases per year, with 1.8 million subjects dying from this malignancy. LC accounts for $11.6 \%$ of total cancer diagnoses and 18.4\% cancer-related deaths (1). Early-stage LC usually does not cause specific symptoms; consequently, more than $70 \%$ LCs are diagnosed at advanced stages $(2,3)$. Recent breakthroughs in targeted and immune therapy resulted in dramatic improvement of LC outcomes $(4,5)$. Nevertheless, LC is a direct cause of death in the majority of patients with this diagnosis (1). LC is often characterized in the scientific literature as a highly aggressive disease; nevertheless, patients diagnosed at stage IA have approximately $80 \%$ probability of being cured (6). Furthermore, 5 -year overall survival rate for stage IA lung cancer may be close to $90 \%$. In contrast, stage IV LC is associated with dismal prognosis, with 5 -year overall survival rate falling below $20 \%$ (7). These data suggest that early diagnosis of LC is a key for reducing mortality from this disease. LC screening studies largely support this hypothesis: for example, it was shown that lowdose computed tomography (LDCT) reduces LC mortality by at least $20 \%$ in persons at-risk (i.e., in smokers) (8-10).

The major problem of LDCT screening is an unacceptably high rate of false-positive findings. Indeed, many healthy people, particularly elderly subjects and smokers, present with so-called lung "nodules", i.e., some lumps detected by X-rays. These lumps reflect topical increase of the density of lung tissue due to alterations in its structure. The most ominous cause of these nodules is the LC. However, many benign processes, e.g., inflammatory infiltrates caused by smoking-related irritation of bronchi, infections, autoimmune processes etc., manifest with lumps which are largely indistinguishable from LC by imaging. Great efforts have been invested to improve radiographic procedures and their interpretation in order to discriminate between LC and non-LC nodules. Besides careful visual analysis of appearance of these nodules, to be performed by highly-trained specialists with the support of various electronic tools, it is suggested to consider some probability factors, e.g., age of the patient and his/her smoking history. This «clinical» approach, although being wise and intuitively attractive, has a high risk of missing malignant disease at a curable stage. Consequently, many subjects with LDCT-detected nodules end up with invasive procedures aimed to obtain a piece of suspected nodule and to subject it to a morphological analysis. Collection of tissue samples from thoracic cavity is obviously associated with suffering of examined subjects and often results in serious complications, particularly bleeding. Unfortunately, even this invasive intervention cannot guarantee right diagnosis: there are many instances of LC, where tissue biopsy produces false-negative results die to actual failure to obtain tumor sample (11-14).

Liang et al. (15) recently presented a test called PulmoSeek, which is based on the detection of LCspecific methylation signatures in circulating tumor DNA 
(ctDNA). They recruited 585 patients from 14 hospitals located in China; these patients had lung nodules with a size ranging from 5 to $30 \mathrm{~mm}$, which were detected by LDCT. All included patients had definite pathologic diagnosis discriminating between LC and benign lesions. Approximately one out of ten plasma DNA samples failed to pass quality control, so the study was focused on 529 patients with informative ctDNA test. $309 \mathrm{ctDNA}$ samples (253 malignant and 56 benign) were used for the methylomic study in order to select the most informative loci. The authors utilized AnchorDx next generation sequencing (NGS) platform for the analysis of methylated cytosines; this platform examines 12,899 genomic regions, which demonstrated LC-specific methylation pattern in a previous study (16), and includes 105,844 CpG sites. Liang et al. (15) identified the most informative regions (which they called features) and utilized a test set consisting of 80 samples (60 malignant and 20 benign) in order to reveal what would be the optimal size of the PulmoSeek assay. They eventually concluded, that 100 the most informative regions is the optimal size for the ctDNA methylation test.

Liang et al. (15) provide thoughtful discussion regarding the requirements for this newly developed assay. They wisely state that it is absolutely inacceptable if the test will miss patients with LC, so they decided to focus on the combination of high sensitivity and high negative predictive value (NPV) instead of considering specificity and positive predictive value. They validated the performance of the PulmoSeek in 140 additional patients (100 LC and 40 nonLC). In this validation cohort PulmoSeek demonstrated 99\% sensitivity and $93 \% \mathrm{NPV}$; when the patients with nodule size ranging from 6 to $20 \mathrm{~mm}$ were considered, both these values achieved $100 \%$. It is important to realize that the ratio between $\mathrm{LC}$ and non-LC patients in this validation set does not reflect "natural" frequency of LC in subjects undergoing LDCT screening. Calculations demonstrate that if the frequency of LC in the studied cohort would be $23 \%$, which is still significantly higher than in real-world patient series, the sensitivity and NPV will both approach to $100 \%$.

Although the performance characteristics of the PulmoSeek are impressive, it is necessary to acknowledge that it delivered erroneous results in a small subset of subjects. The important question, how this series of patients would fare if they were managed by currently available tools. The authors evaluated the performance of Mayo Clinic and Veterans Affairs scores, which integrate imaging characteristics of lung nodules and personal risk factors in order to discriminate between LC and non-LC $(11,13)$. These scoring systems were evidently less reliable as compared to the PulmoSeek with the AUC of 0.843 (0.769$0.918)$ versus AUC of $0.591(0.482-0.688)$ for the Mayo Clinic model and $0.544(0.442-0.640)$ for the Veterans Affairs model.

Patients with suspicious LDCT finding are recommended to undergo PET-CT, as the latter is more informative for differential diagnosis between LC and non-LC. 26 out of 140 patients included in the validation set had PET-CT records. PulmoSeek correctly classified $8 / 10$ patients with solid nodules, 9/11 cases with part-solid nodules and all 5 subjects with ground-glass nodules; these estimates for PET/CT were $6 / 10,7 / 11$ and $0 / 5$, respectively (15).

Several important considerations need to be taken into account while discussing this study. It is clear that the PulmoSeek assay reliably discriminates between LC and non-LC on the level of pathological diagnosis. The current dogma in oncology actually equalizes pathological and clinical diagnosis of cancer disease, so virtually all newly diagnosed malignancies are treated in a rather aggressive way. This attitude relies on the assumption that all detectable cancers will eventually progress if not removed from the body. Screening data obtained for breast cancer suggest that this dogma is not always true. Indeed, breast cancer screening resulted in immediate rise of the number of detected early-stage tumors, but this apparent success was not accompanied by a significant decline of the incidence of metastatic carcinomas. In fact, this effort led to detection of indolent tumors, which would never cause fatal outcome, but largely failed to control potentially lethal cancers $(17,18)$. In any event, right pathological diagnosis is only an intermediate end-point in a cancer screening program, so it is important to ensure that every newly developed approach will indeed eventually result in saved lives.

Many oncological diseases have relatively similar characteristics in patients of different races. This does not apply to lung cancer, which has a number of well-known race-specific features. For example, more than a half of Asian LCs carry EGFR mutations, while this estimate is only around $10 \%$ in people of European race (19-21). $387 / 529$ (73\%) included subjects in the study of Liang et al. (15) were non-smokers, while most of consecutive LC series described in Western countries have significantly higher proportion of smokers. Hence, it is not self-explanatory that PulmoSeek will perform equally well in non-Asian populations. Furthermore, molecular features of smokinginduced and smoking-unrelated LC have very significant 
differences $(5,22)$. It is likely that the performance of NGSbased LC screening tests can be further improved by adjusting to smoking history.

The detection of ctDNA is highly complicated, because it is present in the blood in residual amounts, especially in subjects with early-stage cancers. It is of question, what is the reproducibility of serial blood-takes, i.e., whether a single analysis is sufficient, or if the performance of this assay can be improved by analyzing several samples from the same subject. Surprisingly, relatively little attention has been paid to intrapatient replicability of serial ctDNA tests (23).

Physicians are used to deal with straightforward measurements, which produce single, intuitively understandable numerical or qualitative estimates (consider, for instance, leukocyte count or blood group determination). Many modern assays analyze multiple parameters and use sophisticated, usually patent-protected calculations in order to produce a score. It is often difficult for clinical specialists to sense the genuine meaning of these scores. Furthermore, the results of "omics" assays usually cannot be validated in an independent laboratory, in contrast to, say, pathological diagnosis.

Despite the above limitations, the results obtained by Liang et al. (15) hold a great promise for breakthrough LC management. The PulmoSeek assay certainly deserves integration into clinical practice in the form of wellcontrolled prospective LC screening trial.

\section{Acknowledgments}

Funding: This work was supported by Russian Science Foundation grant \#20-15-00244.

\section{Footnote}

Provenance and Peer Review: This article was commissioned by the editorial office, Translational Lung Cancer Research. The article did not undergo external peer review.

Conflicts of Interest: All authors have completed the ICMJE uniform disclosure form (available at https://dx.doi. org/10.21037/tlcr-21-680). Dr. AGI reports grant from Russian Science Foundation (grant \#20-15-00244). The other authors have no conflicts of interest to declare.

Ethical Statement: The authors are accountable for all aspects of the work in ensuring that questions related to the accuracy or integrity of any part of the work are appropriately investigated and resolved.

Open Access Statement: This is an Open Access article distributed in accordance with the Creative Commons Attribution-NonCommercial-NoDerivs 4.0 International License (CC BY-NC-ND 4.0), which permits the noncommercial replication and distribution of the article with the strict proviso that no changes or edits are made and the original work is properly cited (including links to both the formal publication through the relevant DOI and the license). See: https://creativecommons.org/licenses/by-nc-nd/4.0/.

\section{References}

1. Bray F, Ferlay J, Soerjomataram I, et al. Global cancer statistics 2018: GLOBOCAN estimates of incidence and mortality worldwide for 36 cancers in 185 countries. CA Cancer J Clin 2018;68:394-424.

2. Morgensztern D, Ng SH, Gao F, et al. Trends in stage distribution for patients with non-small cell lung cancer: a National Cancer Database survey. J Thorac Oncol 2010;5:29-33.

3. Lemjabbar-Alaoui H, Hassan OU, Yang YW, et al. Lung cancer: Biology and treatment options. Biochim Biophys Acta 2015;1856:189-210.

4. Nadal E, Massuti B, Dómine M, et al. Immunotherapy with checkpoint inhibitors in non-small cell lung cancer: insights from long-term survivors. Cancer Immunol Immunother 2019;68:341-52.

5. Imyanitov EN, Iyevleva AG, Levchenko EV. Molecular testing and targeted therapy for non-small cell lung cancer: Current status and perspectives. Crit Rev Oncol Hematol 2021;157:103194.

6. Maeda R, Yoshida J, Ishii G, et al. Long-term outcome and late recurrence in patients with completely resected stage IA non-small cell lung cancer. J Thorac Oncol 2010;5:1246-50.

7. Goldstraw P, Chansky K, Crowley J, et al. The IASLC Lung Cancer Staging Project: Proposals for Revision of the TNM Stage Groupings in the Forthcoming (Eighth) Edition of the TNM Classification for Lung Cancer. J Thorac Oncol 2016;11:39-51.

8. National Lung Screening Trial Research Team; Aberle DR, Adams AM, et al. Reduced lung-cancer mortality with low-dose computed tomographic screening. $\mathrm{N}$ Engl J Med 2011;365:395-409.

9. de Koning HJ, van der Aalst CM, de Jong PA, et al. Reduced Lung-Cancer Mortality with Volume CT 
Screening in a Randomized Trial. N Engl J Med 2020;382:503-13.

10. Thai AA, Solomon BJ, Sequist LV, et al. Lung cancer. Lancet 2021;398:535-54.

11. Swensen SJ, Silverstein MD, Ilstrup DM, et al. The probability of malignancy in solitary pulmonary nodules. Application to small radiologically indeterminate nodules. Arch Intern Med 1997;157:849-55.

12. Diederich S, Das M. Solitary pulmonary nodule: detection and management. Cancer Imaging 2006;6:S42-6.

13. Gould MK, Ananth L, Barnett PG, et al. A clinical model to estimate the pretest probability of lung cancer in patients with solitary pulmonary nodules. Chest 2007;131:383-8.

14. McWilliams A, Tammemagi MC, Mayo JR, et al. Probability of cancer in pulmonary nodules detected on first screening CT. N Engl J Med 2013;369:910-9.

15. Liang W, Chen Z, Li C, et al. Accurate diagnosis of pulmonary nodules using a noninvasive DNA methylation test. J Clin Invest 2021;131:145973.

16. Liang $W$, Zhao $Y$, Huang $W$, et al. Non-invasive diagnosis of early-stage lung cancer using high-throughput targeted DNA methylation sequencing of circulating tumor DNA (ctDNA). Theranostics 2019;9:2056-70.

Cite this article as: Kuligina ES, Iyevleva AG, Imyanitov EN. Integration of the blood test into the low-dose computed tomography lung cancer screening: reliable discrimination between malignant and non-malignant radiographic findings. Transl Lung Cancer Res 2021;10(10):4035-4038. doi: 10.21037/ tlcr-21-680
17. Welch HG, Prorok PC, O'Malley AJ, et al. Breast-Cancer Tumor Size, Overdiagnosis, and Mammography Screening Effectiveness. N Engl J Med 2016;375:1438-47.

18. Lannin DR, Wang S. Are Small Breast Cancers Good because They Are Small or Small because They Are Good? N Engl J Med 2017;376:2286-91.

19. Duster T. Medicalisation of race. Lancet 2007;369:702-4.

20. Zhou W, Christiani DC. East meets West: ethnic differences in epidemiology and clinical behaviors of lung cancer between East Asians and Caucasians. Chin J Cancer 2011;30:287-92.

21. Chapman AM, Sun KY, Ruestow P, et al. Lung cancer mutation profile of EGFR, ALK, and KRAS: Metaanalysis and comparison of never and ever smokers. Lung Cancer 2016;102:122-34.

22. Smolle E, Pichler M. Non-Smoking-Associated Lung Cancer: A distinct Entity in Terms of Tumor Biology, Patient Characteristics and Impact of Hereditary Cancer Predisposition. Cancers (Basel) 2019;11:204.

23. Kuligina ES, Meerovich R, Zagorodnev KA, et al. Content of circulating tumor DNA depends on the tumor type and the dynamics of tumor size, but is not influenced significantly by physical exercise, time of the day or recent meal. Cancer Genet 2021;256-257:165-78. 